\title{
ON THE NUMBER OF LIMIT CYCLES IN PIECEWISE LINEAR LIÉNARD SYSTEMS
}

\author{
A. Tonnelier \\ Cortex Project, INRIA Lorraine \\ Campus Scientifique, \\ 54 602 Villers-lès-Nancy, \\ FRANCE
}

\begin{abstract}
In a previous paper [Tonnelier, 2002] we conjectured that a Liénard system of the form $\dot{x}=p(x)-y, \dot{y}=x$ where $p$ is piecewise linear on $n+1$ intervals has up to $2 n$ limit cycles. We construct here a general class of functions $p$ satisfying this conjecture. Limit cycles are obtained from the bifurcation of the linear center.
\end{abstract}

Keywords: Limit cycles, piecewise linear systems.

\section{Introduction and Main Result}

A number of physical systems controlled by switching actions cannot be described with smooth dynamical systems. Many applications in different part of sciences are modeled by piecewise linear systems of ordinary differential equations. Examples come from control engineering, design of electrical circuits, embedded software, mechanical systems. The modeling of systems that operate in different linear modes with a transition between modes idealized as an instantaneous one leads to piecewise linear differential equations. In mechanical systems, for instance, dry friction, stick-slip oscillations and impact are described with non-smooth dynamical systems (see e.g. [Leine et al., 2000]).

On the other hand, piecewise linear functions are introduced as an approximation or a global linearization of a nonlinear dynamics. Taking the advantage of their linear parts, piecewise linear systems lead to analytic calculations and provide a better understanding of the richness of the nonlinear 
dynamics. Starting from linear systems, nonlinear dynamics are captured quite naturally by working with piecewise linear equations. In neuroscience, for instance, the subthreshold and superthreshold behavior of the neuron is well approximate by a piecewise linear regime introduced as a generalization of the integrate-and-fire model [Tonnelier \& Gerstner, 2003].

The theory of smooth dynamical systems is the subject of numerous works, see for instance [Guckenheimer and Holmes, 1983], [Kuznetsov, 1998]. Since the pioneering works of Filippov [1964] and Andronov et al. [1966], the mathematical study of non-smooth dynamical systems have been developed. More recent results can be found in [Aubin \& Cellina, 1984; Kunze, 2000]. One of the most difficult problem in the qualitative study of dynamical systems is the question of the number and the location of limit cycles. One expects that results from piecewise linear systems can lead to insights into this problem. Most studies deal with planar systems with a limited number of zones. The bifurcation set of continuous piecewise linear systems with two zones and three zones have been studied in details [Freire et al., 1998; Freire et al., 1999; Freire et al., 2002]. A complete study of systems with a line of discontinuity is performed in [Giannakopoulos \& Pliete, 2001]. In [Leine et al., 2000] authors studied bifurcations in discontinuous dynamical systems. There are few analytical results on systems with a large number of zones and little is known about their dynamics. During the writing of this paper, two works on this topic have been published [Llibre et al., 2003; Llibre \& Ponce, 2003]. In the former, the Poincaré-Bendixson theory is used and in the latter averaging theorem is used that seems computationally equivalent to the approach followed in the present paper. Given the difficulty of the general problem, we will restrict our attention to the so-called Liénard system:

$$
\begin{aligned}
& \dot{x}=\epsilon p(x)-y, \\
& \dot{y}=x,
\end{aligned}
$$

where $(x, y) \in R^{2}, p$ is a piecewise linear function and $\epsilon$ is a parameter that we consider for convenience. The phase plane is divided into zones so that the restriction of the system into each zone is a linear system with constant coefficients. The boundaries between zones are parallel straight lines given by the discontinuity points of the piecewise linear function $p$. One expects that the number of limit cycles is closely related to the number of zones, that is the number of parts of the phase plane where system (1) is linear. In [Tonnelier, 2002] we proposed the two conjectures

Conjecture 1 The piecewise linear Liénard system (1) with $n+1$ zones has up to 2 limit cycles. 
Conjecture 2 The continuous piecewise linear Liénard system (1) with $n+1$ zones has up to $n$ limit cycles.

Let us discuss the simplest case where $n=1$. The proof of conjecture 1 goes back to Andronov et al. (see [Giannakopoulos \& Pliete, 2001 ] for a complete treatment) and conjecture 2 is already stated in [Lum \& Chua, 1992] (see [Freire et al., 1998] for a proof). Note also that the case $n=2$ is related to the McKean caricature of the FitzHugh-Nagumo equation [McKean, 1970]. For $n=2$, conjecture 2 is treated in [Freire et al., 2002]. For a large number of zones, a piecewise continuous Liénard system with $2 n+3$ zones and $n$ limit cycles has been proposed [Llibre \& Ponce, 2003].

In this paper we provide a general class of systems satisfying the first conjecture. Moreover, we give a simple recursive algorithm to generate this class of functions. The main result of our paper is the following one:

Theorem 1 Let $n$ be a positive integer and $p$ the piecewise linear function (see Fig. 1)

$$
p(x)=-x+\mu_{i} \text { if } x \in\left(a_{i}, a_{i+1}\right), i \in\{0, \ldots, n\},
$$

where $\left(a_{0}=-\infty\right)<0<a_{1}<\ldots<a_{n}\left(<a_{n+1}=+\infty\right)$ and $\left(\mu_{i}\right)_{i=0 . . n}$, are two finite sequences of real numbers. For $\epsilon \neq 0$ sufficiently small, there exist sequences $\left(a_{i}\right)$ and $\left(\mu_{i}\right)$ such that system (1) admits $2 n$ limit cycles.

The change of variable $\tilde{y}=y-\mu_{0} \epsilon$ enables us to consider the case $\mu_{0}=0$. Note that $p$ is discontinuous at the points $\left(a_{i}\right)$ and has a jump of discontinuity equal to $\mu_{i}-\mu_{i-1}$. It should be noticed that ordinary differential equations with discontinuous right-hand side can lead to trajectories that intersect the set of discontinuity and remain on it. These so-called sliding solutions are possible when $\mu_{i+1}<\mu_{i}$. However, as we will show, the existence of $2 n$ limit cycles requires that the $\mu_{i}$ determine a non-decreasing sequence. Then sliding solutions do not appear in our study and solutions of (1) are defined in a classical manner.

The paper is organized as follows. In section 2 we introduce the mathematical tools needed to state and prove Theorem 1. We also provide a recursive algorithm to generate a family of functions satisfying Theorem 1 . Section 3 is devoted to a discussion.

\section{A Piecewise Linear Perturbation of the Lin- ear Center}

Melnikov's theory has proved to be a powerful tool for predicting the number, positions and multiplicities of limit cycles that bifurcate from homoclinic and 
heteroclinic orbits under perturbations [Guckenheimer \& Holmes, 1983]. It has been successfully applied for the occurrence of bifurcations in physical systems (see references in [Sanjuán, 1998]). Basically, the theory associates a function to a given dynamical system. The roots of this function are related to the existence and location of limit cycles. Then, the differential problem is reduced to an algebraic one that corresponds to a search for roots of a function.

\subsection{Melnikov theory}

The Melnikov theory have been developed for the study of planar systems of the form

$$
\dot{u}=f(u)+\epsilon g(u)
$$

where $u \in R^{2}, \epsilon \ll 1$ and $f, g$ are sufficiently smooth functions. It is assumed that the unperturbed system, i.e. $\epsilon=0$, has a one-parameter family of periodic solutions $\Gamma_{r}: \gamma_{r}(t), r>0$ with period $T_{r}$. The Melnikov function for system (3) is given by

$$
M(r)=\int_{0}^{T_{r}} e^{-\int_{0}^{t} \nabla f\left(\gamma_{r}(s)\right) d s} f \wedge g\left(\gamma_{r}(t)\right) d t
$$

where the wedge product of $u, v \in R^{2}$ is defined by $u \wedge v=u_{1} v_{2}-v_{1} u_{2}$. The following theorem is proved in [Blows \& Perko, 1994] (see also Guckenheimer \& Holmes, 1983),

Theorem 2 If there exists $r_{1}, r_{2}, \ldots, r_{n}$ such that $M\left(r_{j}\right)=0$ and $M^{\prime}\left(r_{j}\right) \neq$ $0, j=1, \ldots, n$, then for all sufficiently small $\epsilon \neq 0$ system (3) has $n$ hyperbolic limit cycles in an $O(\epsilon)$ neighborhood of $\Gamma_{r_{j}}$ that bifurcate from the periodic orbits $\gamma_{r_{j}}(t)$. Furthermore if $M\left(r_{0}\right) \neq 0$, then for all sufficiently small $\epsilon \neq 0$, system (3) has no cycle in an $O(\epsilon)$ neighborhood of $\Gamma_{r_{0}}$.

\subsection{The Melnikov function of the piecewise linear Liénard system}

For small $\epsilon$, Eq. (1) is a perturbation of the linear center $\dot{x}=-y, \dot{y}=x$ that has a one-parameter family of periodic orbits $\Gamma_{r}:(r \cos t, r \sin t), r>0$, with a period $T_{r}=2 \pi$. Note that system (1) is invariant under the change of variables $(t, x, y, \epsilon) \rightarrow(-t, x,-y,-\epsilon)$. Therefore the stability of limit cycles

changes with the sign of $\epsilon$. Note also that the origin is the only fixed point of the system and it is easy to check that the origin is an unstable focus when $\epsilon<0$. 
Using $\nabla f(u)=0$, the Melnikov function (4) of the Liénard system (1) is given by

$$
M(r)=\int_{0}^{2 \pi}-r \cos t p(r \cos t) d t
$$

Using the change of variable $t \rightarrow 2 \pi-t$ in the interval $[\pi, 2 \pi]$, we have

$$
M(r)=2 \int_{0}^{\pi}-r \cos t p(r \cos t) d t
$$

Let $r>0$ and $q \in\{0, \ldots, n\}$ be the integer such that $a_{q}<r<a_{q+1}$. We define $t_{i}, i \in\{0, \ldots, q+1\}$, the decreasing finite sequence given by $t_{0}=\pi$,

$$
\cos t_{i}=\frac{a_{i}}{r}
$$

for $i=1, \ldots, q$ and $t_{q+1}=0$. On $\left(t_{i+1}, t_{i}\right)$, we have $p(r \cos t)=-r \cos t+\mu_{i}$. Therefore we obtain

$$
M(r)=2 r^{2} \int_{0}^{\pi} \cos ^{2} t d t-2 r \sum_{i=0}^{q} \mu_{i} \int_{t_{i+1}}^{t_{i}} \cos t d t .
$$

Computing the integrals, we obtain the following:

$$
M(r)=\pi r^{2}-2 \sum_{i=1}^{q-1} \mu_{i}\left(\sqrt{r^{2}-a_{i}^{2}}-\sqrt{r^{2}-a_{i+1}^{2}}\right)-2 \mu_{q} \sqrt{r^{2}-a_{q}^{2}} .
$$

Let us recall that the previous expression is derived for $a_{q}<r<a_{q+1}$. Let $r>0$, the Melnikov function $M$ is written

$$
M(r)=\pi r^{2}-2 \sum_{i=1}^{i=n} d_{i} \sqrt{r^{2}-a_{i}^{2}} H\left(r-a_{i}\right)
$$

where $d_{i}=\mu_{i}-\mu_{i-1}$ for $i \in\{1, \ldots, n\}$ and $H$ is the Heaviside step function $H(x)=0$ if $x<0$ and $H(x)=1$ if $x>0$. We note that $M(r)$ is continuous but not $C^{1}\left(R^{+}\right)$.

\subsection{Proof of Theorem 1}

Checking the proof of Theorem 2 in [Blows and Perko, 1994], we can see that smoothness is required for $f$ but the discontinuity of $g$ at isolated values is not a restriction for the use of Theorem 2. From Theorem 2 and the expression (5) of the Melnikov function associated with the Liénard system (1),(2), the following proposition holds. 
Proposition 1 The Liénard system (1),(2) has exactly p hyperbolic limit cycles asymptotic to circles of radii $r_{j}$ as $\epsilon \rightarrow 0$ if and only if the function

$$
f_{n}\left(r^{2}\right)=\pi r^{2}-2 \sum_{i=1}^{i=n} d_{i} \sqrt{r^{2}-a_{i}^{2}} H\left(r^{2}-a_{i}^{2}\right)
$$

has $p$ positives roots $r=r_{j}$ such that $f_{n}^{\prime}\left(r_{j}^{2}\right) \neq 0$.

More specifically, to prove Theorem 1, we will show in the following that $f_{n}$ has up to $2 n$ roots. For $n=3$, the typical shape of the Melnikov function with three pairs of roots is shown in Fig. 2A.

Proof. We note $x=r^{2}$ and we define the family of functions:

$$
f_{k}(x)=\pi x-2 \sum_{i=1}^{i=k} d_{i} \sqrt{x-a_{i}^{2}} H\left(x-a_{i}^{2}\right), \quad k=1, \ldots, n
$$

Note that $f_{k}$ and $f_{k+1}$ coincide on $\left(0, a_{k+1}\right)$. We construct recursively the function $f_{k}$, and thus $p$, choosing appropriate values for $\mu_{k}$ and $a_{k}$ in order to ensure, at each step, the existence of two additional roots of $f_{k}$ in $\left(a_{k},+\infty\right)$.

Let us start with $k=1$. We have

$$
f_{1}(x)=\pi x-2 d_{1} \sqrt{x-a_{1}^{2}}
$$

for $x>a_{1}^{2}$. It is easy to show that the equation $f_{1}(x)=0$ has two real solutions if and only if $d_{1}>\pi a_{1}$. We easily check that these solutions are greater than $a_{1}^{2}$. Note that $a_{1}>0$ is arbitrary chosen.

In order to start the recurrence, we consider the case $k=2$. We choose $a_{2}$ sufficiently big such that $a_{2}^{2}$ is greater than the two roots of $f_{1}$. This requirement ensures that the two roots of $f_{1}$ are also roots of $f_{2}$. Note that we have $f_{2}\left(a_{2}^{2}\right)=f_{1}\left(a_{2}^{2}\right)>0$. We take $d_{2}$ such that $\exists x^{*}>a_{2}^{2}, f_{2}\left(x^{*}\right)<0$. Notice that this choice is always possible since for $x>a_{2}^{2}$ and fixed, $\lim _{d_{2} \rightarrow \infty} f_{2}(x)<$ 0 (note that $f_{2}\left(a_{2}^{2}\right)$ does not depend on $d_{2}$ ). Using $\lim _{x \rightarrow \infty} f_{2}(x)=+\infty$ and the continuity of $f_{2}$ on $\left(a_{2}^{2},+\infty\right)$ it is clear that $f_{2}$ has two additional roots in $\left(a_{2}^{2},+\infty\right)$.

We assume that we have constructed two finite sequences $a_{1}, \ldots, a_{k-1}$ and $\mu_{1}, \ldots, \mu_{k-1}$ such that $f_{k-1}$ has $2 k-2$ roots on $\left(a_{1}^{2},+\infty\right)$. Then we choose $a_{k}^{2}$ sufficiently big to be greater than the roots of $f_{k-1}$. Then $f_{k}$ has $2 k-2$ roots in $\left(0, a_{k}\right)$. Moreover we require $f_{k}\left(a_{k}^{2}\right)>0$. We choose $d_{k}>0$ sufficiently big to ensure the existence of $x^{*}>a_{k}^{2}$ such that $f_{k}\left(x^{*}\right)<0$. Since $\lim _{x \rightarrow \infty} f_{k}(x)=+\infty$ it follows that $f_{k}$ has two additional roots in $\left(a_{k},+\infty\right)$. 
If we study the variations of $f_{k}, k \in\{1, \ldots, n\}$, we can refine the proposition and show that $2 n$ is the maximal number of limit cycles. Moreover, if we note indifferently $x_{k}$ the two roots of $f_{k}$ in $\left(a_{k}, a_{k+1}\right)$, by construction we have $f_{n}^{\prime}\left(x_{k}\right)=f_{k}^{\prime}\left(x_{k}\right) \neq 0$ and then the limit cycles are hyperbolic.

\subsection{Some specific examples}

In this section we derive explicit sufficient conditions on $\left(a_{k}\right)$ and $\left(\mu_{k}\right)$ for the existence of $2 n$ limit cycles. Note that shifting the $y$-axis in (1), we can take $\mu_{0}=0$. A recursive construction of the function $p$ is provided using the following proposition

Proposition 2 If $p$ is such that $\left(a_{k}\right)$ and $\left(\mu_{k}\right)$ satisfy

$$
a_{1}>0, \mu_{1}>\pi a_{1}, a_{k} \geq \frac{2 \mu_{k-1}}{\pi}, \mu_{k} \geq \pi a_{k}, k=\{2, \ldots, n\}
$$

then system (1),(2) has 2 n hyperbolic limit cycles.

Proof. Let us recall that the equation $f_{1}(x)=\pi x-2 \mu_{1} \sqrt{x-a_{1}^{2}}=0$ has two distinct real solutions on $\left(a_{1}^{2},+\infty\right)$ if and only if $\mu_{1}>\pi a_{1}$ (i.e. $d_{1}>\pi a_{1}$ ). Moreover these two solutions are given by

$$
x_{ \pm}^{2}=\frac{2 \mu_{1}^{2} \pm 2 \mu_{1} \sqrt{\mu_{1}^{2}-\pi^{2} a_{1}^{2}}}{\pi^{2}} .
$$

Hence, it is sufficient to choose $a_{2}$ such that $a_{2} \geq 2 \mu_{1} / \pi$. For $d_{2}>0$, the study of the derivative of $f_{2}$ shows that there exists $x_{2}^{*}$ such that $f_{2}$ is decreasing on $\left(a_{2}^{2}, x_{2}^{*}\right)$ and increasing on $\left(x_{2}^{*},+\infty\right)$ (note that $d_{2}<0$, i.e. $\mu_{2}<\mu_{1}$, does not allow the existence of roots of $f_{2}$ in $\left.\left(a_{2}^{2},+\infty\right)\right)$. Then $f_{2}$ has at most two solutions on $\left(a_{2}^{2}, a_{3}^{2}\right)$. We have

$$
\begin{aligned}
f_{2}(x) & =\pi x-2 \mu_{1} \sqrt{x-a_{1}^{2}}-2\left(\mu_{2}-\mu_{1}\right) \sqrt{x-a_{2}^{2}} \\
& <\pi x-2 \mu_{1} \sqrt{x-a_{2}^{2}}-2\left(\mu_{2}-\mu_{1}\right) \sqrt{x-a_{2}^{2}} .
\end{aligned}
$$

Then the following (strict) inequality holds

$$
f_{2}(x)<\pi x-2 \mu_{2} \sqrt{x-a_{2}^{2}}
$$

Since $\pi x-2 \mu_{2} \sqrt{x-a_{2}^{2}}$ has two real roots (greater than $a_{2}^{2}$ ) if $\mu_{2}>\pi a_{2}$, we conclude that a sufficient condition to have two roots in $\left(a_{2}^{2},+\infty\right)$ is $\mu_{2} \geq \pi a_{2}$ 
(in particular, $\mu_{2}>\mu_{1}$ ). Now, the next step is to choose $a_{3}^{2}$ greater than the roots of $f_{2}$. Noting that

$$
f_{2}(x)>\pi x-2 \mu_{1} \sqrt{x-a_{1}^{2}}-2\left(\mu_{2}-\mu_{1}\right) \sqrt{x-a_{1}^{2}}=\pi x-2 \mu_{2} \sqrt{x-a_{1}^{2}}
$$

it is clear that the two roots of $f_{2}$ on $\left(a_{2}^{2},+\infty\right)$ are between the roots of $\pi x-2 \mu_{2} \sqrt{x-a_{1}^{2}}$. Thus, we choose $a_{3} \geq 2 \mu_{2} / \pi$, i.e. $a_{3}^{2}$ is greater than the roots of $\pi x-2 \mu_{2} \sqrt{x-a_{1}^{2}}$. Note that $f_{4}\left(a_{3}^{2}\right)>0$.

The study on the intervals $\left(a_{3}^{2}, a_{4}^{2}\right), \ldots,\left(a_{k-1}^{2}, a_{k}^{2}\right)$ is very similar and it is not difficult to prove that a sufficient condition for the existence of $2 k$ roots for $f_{k}$ is given by $a_{k} \geq 2 \mu_{k-1} / \pi$ and $\mu_{k} \geq \pi a_{k}$.

The following sequence is an example that fulfilled the conditions of Proposition 2 :

$$
a_{1}>0, \alpha>1, a_{k}=r a_{1} 2^{k-1}, \mu_{k}=\alpha a_{1} \pi 2^{k-1} .
$$

In table 1 we numerically compute the location of the limit cycles for $a_{1}=1$ and $\alpha=2$, i.e. $a_{k}=2^{k}, \mu_{k}=\pi 2^{k}$. Figure 3 shows, in the phase plane, three pairs of limit cycles of the Liénard system obtained for $\epsilon=0.01$.

\section{Discussion}

Since they frequently appear in physical and biological systems, limit cycles have attracted a wide attention. Limit cycles model self sustained oscillations that are involved in numerous applications. The problem of finding the number and the location of limit cycles is related to the already unsolved Hilbert's 16th problem. Piecewise linear systems capture the richness of the dynamical behavior of general nonlinear systems, including limit cycles. Taking the advantage of their linear parts, one expects to obtain insights into the problem of the existence of limit cycles.

In this paper we have studied the number and the location of limit cycles of a piecewise linear Liénard system. Given an even integer $2 n$, we construct a planar piecewise linear system with $2 n$ limit cycles. A piecewise linear differential system with an arbitrary number of limit cycles has already been proposed [Llibre \& Ponce, 2003]. We give here a general class of functions and present a recursive algorithm to generate it. Moreover we claim that our system is minimal in the sense that a system with lesser linear parts, i.e. lesser than $n+1$, has lesser limit cycles. Moreover, based on numerical simulations, we find that we also provide an example for Conjecture 2 taking a continuous version of the function $p$ with sufficiently small matching zones. 


\section{Acknowledgments}

We thank Hubert Meignen for many valuable suggestions. We thank the laboratories of J. Demongeot (TIMC, IMAG) and W. Gerstner (LCN, EPFL) for their hospitality and support.

\section{References}

Andronov, A., Vitt, A. \& Khaikin, S. [1966] Theory of oscillations (Pergamon Press, Oxford).

Aubin, J. P. \& Cellina, A. [1984] Differential Inclusions (Springer-Verlag, Berlin).

Blows, T. R. \& Perko, L. M. [1994] "Bifurcation of limit cycles from centers and separatrix cycles of planar analytic systems," SIAM Review 36(3), 341-376.

Filippov, A. F. [1964] "Differential equations with discontinuous righthand side," Amer. Math. Soc. Translations Series 42(2), 199-231.

Freire, E., Ponce, E., Rodrigo, F. \& Torres, F. [1998] "Bifurcation sets of continuous piecewise linear systems with two zones," Int. J. Bifurcation and Chaos 8(11), 2073-2097.

Freire, E., Ponce, E. \& Ros, J. [1999] "Limit cycle bifurcation from center in symmetric piecewise-linear systems," Int. J. Bifurcation and Chaos 9(5), 895-907.

Freire, E., Ponce, E., Rodrigo, F. \& Torres, F. [2002] "Bifurcation sets of symmetrical continuous piecewise linear systems with three zones," Int. J. Bifurcation and Chaos 12(8), 1675-1702.

Giannakopoulos, F. \& Pliete, K. [2001] "Planar systems of piecewise linear differential equations with a line of discontinuity," Nonlinearity 14, 1611-1632.

Guckenheimer, J. \& Holmes, P. J. [1983] Nonlinear oscillations, Dynamical Systems and Bifurcations of Vector Fields (Springer-Verlag, New York).

Kunze, M. [2000] Non-Smooth Dynamical Systems (Springer-Verlag, Berlin).

Kuznetsov, Y. [1998] Elements of Applied Bifurcation Theory (SpringerVerlag, New York). 
Leine, R. I., Van Campen, D. H. \& Van de Vrande, B. L. [2000] "Bifurcations in nonlinear discontinuous systems," Nonlinear Dynamics 23, 105-164.

Llibre, J. \& Ponce, E. [2003] "Piecewise linear feedback systems with arbitrary number of limit cycles," Int. J. Bifurcation and Chaos 13(4), 895-904.

Llibre, J., Ponce, E. \& X. Zhang [2003] "Existence of piecewise linear differential systems with exactly $n$ limit cycles for all $n \in \mathcal{N}, "$ Nonlinear Analysis 54, 977-994.

Lum, R. \& Chua, L. O. [1992] "Generic properties of continuous piecewise linear vector field in $R^{2}$," IEEE Trans. Circuits Syst. 38(2), 10431066.

McKean, M. P. [1970] "Nagumo's equation," Advances in Mathematics 4, 209-223.

Sanjuán, M. A. F. [1998] "Liénard systems, limit cycles, and Melnikov theory," Phys. Rev. E 57(1), 340-344.

Tonnelier, A. [2002] "The McKean's caricature of the FitzHugh-Nagumo model. I : The space-clamped system," SIAM J. Appl. Math. 63, 459-484.

Tonnelier, A. \& Gerstner, W. [2003] "Piecewise linear differential equations and integrate-and-fire neurons: insights from two-dimensional membrane models," Phys. Rev. E 67(021908). 


\section{Table}

Table 1. Values of the radius of circles that approximate the limit cycles of the Liénard system as $\epsilon \rightarrow 0$. These values are obtained as the two roots $r_{k, 1}, r_{k, 2}$ of $f_{n}$ (see proposition 1 ) in $\left(a_{k}, a_{k+1}\right), k \in\{1, \ldots, n\}$ for $n=7$ discontinuities and $a_{1}=1, a_{k}=2^{k}$ and $\mu_{k}=\pi 2^{k}$.

\begin{tabular}{cccccccc}
\hline $\mathrm{k}$ & 1 & 2 & 3 & 4 & 5 & 6 & 7 \\
\hline$a_{k}$ & 1 & 4 & 8 & 16 & 32 & 64 & 128 \\
$r_{k, 1}$ & 1.1882 & 4.3147 & 8.0432 & 16.0534 & 32.1003 & 64.1990 & 128.3978 \\
$r_{k, 2}$ & 1.8515 & 6.9042 & 14.4240 & 28.9705 & 57.9707 & 115.9489 & 231.8997 \\
\hline
\end{tabular}




\section{Figure Legends}

Figure 1: Graph of the piecewise linear function $p$ with discontinuities at $a_{1}, a_{2}, \ldots, a_{n}$.

Figure 2: (A) Graph of the Melnikov function for the Liénard system (1) where the function $p$ is shown in panel (B). The nonzero roots of the Melnikov function correspond to three pairs (unstable and stable) of limit cycles. Parameters are those of Table 1.

Figure 3: Numerical computation of limit cycles in the phase plane of the Liénard system $\dot{x}=\epsilon p(x)-y, \dot{y}=x$ for $\epsilon=0.01$ and $p$ given in Table 1 (we restrict here to three discontinuities). The locations of the limit cycles are predicted by the roots of the Melnikov function given in Fig.2A. The full lines represent stable limit cycles and the dashed line represent unstable limit cycles. 


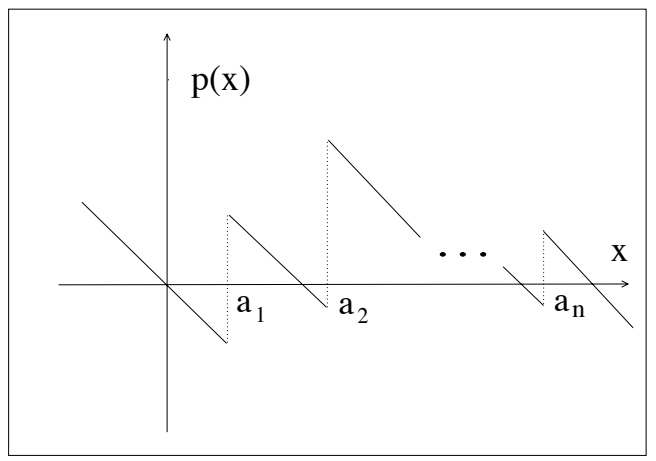

Figure 1. 

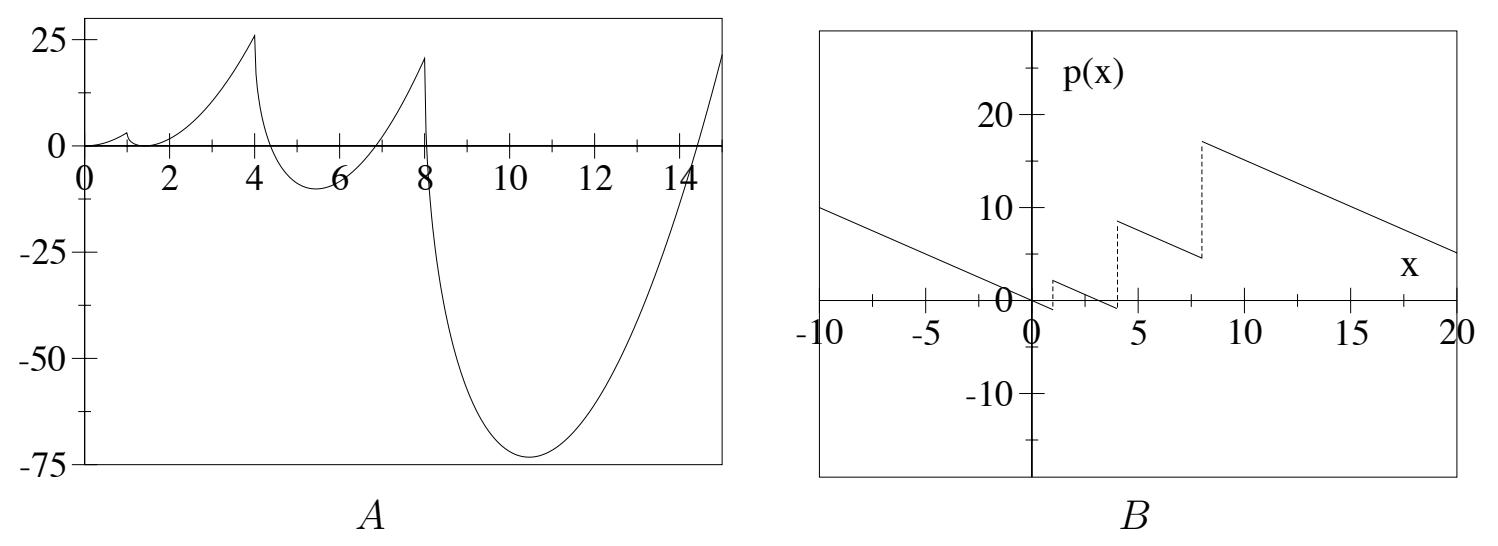

Figure 2. 


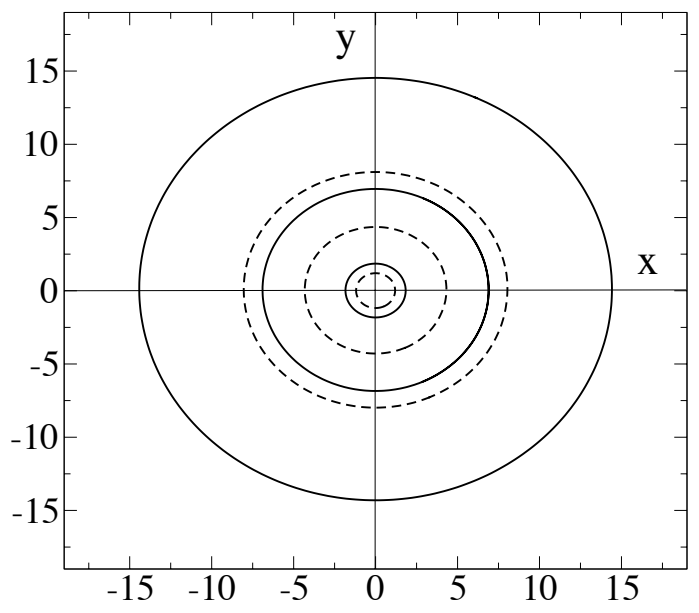

Figure 3. 